\title{
Cordial Labelling Of K-Regular Bipartite Graphs for $K=1,2$, N, N-1 Where K Is Cardinality of Each Bipartition
}

\author{
Pranali Sapre \\ JJT University, Jhunjhunu, Rajasthan, India \\ Assistant Professor,Vidyavardhini's College of Engineering \& Technology \\ Vasai Road Dist. Thane. Maharashtra
}

Abstract: In the labelling of graphs one of the types is cordial labelling. In this we label the vertices 0 or 1 and then every edge will have a label 0 or 1 if the end vertices of the edge have same or different labellings respectively. Here we are going find whether a k-regular bipartite graph can be cordial for different values of $k$.

\section{Introduction :}

- Regular graph: A graph G is said to be a regular graph if degree of each vertex a same. It is called k-regular if degree of each vertex is $\mathrm{k}$.

- Bipartite graph: Let $\mathrm{G}$ be a graph. If the vertex of $\mathrm{G}$ is divided into two subsets $\mathrm{A}$ and $\mathrm{B}$ such that there is no edge 'ab' with $a, b \in A$ or $a, b \in B$ then $\mathrm{G}$ is said to be bipartite that is, Every edge of $\mathrm{G}$ joins a
vertex in
A to
a
vertex
in
B.

The sets A and B are called partite sets of G.

- Complete graph: A graph in which every vertex is adjacent to every other vertex is a complete graph. For a complete graph on $n$ vertices degree of each vertex is $n-1$.

- Complete bipartite graph: A bipartite graph $\mathrm{G}$ with bipartition ( A,B ) is said to be complete bipartite if every vertex in A is adjacent to every vertex in B and vice versa.

- Cycle: A closed path is called a cycle.

- Labelling of a graph:

Vertex labelling :It is a mapping from set of vertices to set of natural numbers .

Edge labelling :It is a mapping from set of edges to set of natural numbers .

- Cordial labelling: For a given graph $\mathrm{G}$ label the vertices of $\mathrm{G}$ ' 0 ' or ' 1 '. And every edge ' $a b$ ' of $\mathrm{G}$ will be labeled as ' 0 ' if the labeling of the vertices ' $a$ ' and ' $b$ ' are same and will be labeled as ' 1 ' if the labeling of the vertices ' $a$ ' and ' $b$ ' are different. Then this labeling is called a "cordial labeling” or the graph $G$ is called a "cordial graph" iff,

$$
\begin{aligned}
& \text { |number of vertices labeled ' } 0 \text { '-number of vertives labeled ' } 1 \mid \leq 1 \\
& \mid \text { number of edges labeled ' } 0 \text { '-number of edges labeled ' } 1 \mid \leq 1
\end{aligned}
$$

Theorem 1. If $\mathrm{G}$ is a 1- regular bipartite graph with partite sets $\mathrm{A}$ and $\mathrm{B}$ with $\mathrm{G}$ is cordial iff $\mathrm{n}=0,1,3 \bmod 4$.

$$
|\mathrm{A}|=|\mathrm{B}|=\mathrm{n} \quad \text { Then }
$$

\section{Case $I . ~ n=4 m$} like,

$$
\text { Let } m=1 \text { i.e. } n=4 \text { then } G \text { looks }
$$

0

0

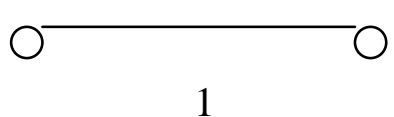

0

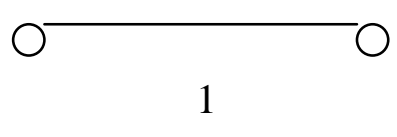

1

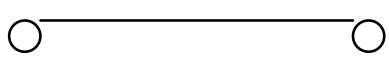

0

1

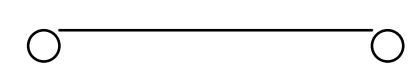


This can be labeled as shown is figure. This is CORDIAL.

Now for $n=4 m$ for $m>1$ ' $G$ ' can be considered as combination of graph shown above and hence can be labeled repeatedly as above which is CORDIAL.

Hence,

$$
\text { G IS CORDIAL FOR } \mathrm{n}=4 \mathrm{~m}
$$

\section{Case II. $n=4 \mathrm{~m}+1$}

Consider $\quad n=4 m+1 \quad$ for $m \in Z$

Consider any edge 'uv' $\in \mathrm{E}(\mathrm{G})$, for $u \in A, v \in B$

Then $\mathrm{G}-\{\mathrm{uv}\}$ is a bipartite graph with $\mathrm{n}=4 \mathrm{~m}$. Hence it has a cordial labeling as given in case ( $\mathrm{i}$ ) which gives,

|\# of edges labeled ' 0 ' - \# of edges labeled ' 1 ' $\mid=0$ hence along with the same labeling if we label " $u$ " as ' 0 ' and " $v$ " as ' 1 ' we get,

| \# of vertices labeled ' 0 '- \# of vertices labeled ' 1 ' $\mid=0$ and

|\# of edges labeled ' 0 '- \# of edges labeled ' 1 ' $\mid=1$

Hence it is cordial.

\section{G IS CORDIAL FOR $n=4 m+1$}

\section{Case III. Let $n=4 \mathrm{~m}+2$}

Consider $n=4 m+2 \quad$ for $m \in Z$

$|\mathrm{A}|=|\mathrm{B}|=4 \mathrm{~m}+2$

Assume $\mathrm{G}$ is cordial.

Let $\alpha_{0}$ be the number of vertices in A labeled ' 0 '

Let $\alpha_{1}$ be the number of vertices in A labeled ' 1 ',

Let $\beta_{0}$ be the number of vertices in B labeled ' 0 ',

Let $\beta_{1}$ be the number of vertices in B labeled ' 1 ',

$\therefore$ Total number of edges 'ab' where 'a' is labeled 0 and $b \in B=\alpha_{0}$

Total number of edges 'ab' where ' $\mathrm{b}$ ' is labeled 0 and $a \in A=\beta_{0}$

Total number of edges 'ab' where 'a' is labeled 1 and $b \in B=\alpha_{1}$

Total number of edges ' $a b$ ' where ' $b$ ' is labeled 1 and $a \in A=\beta_{1}$

Let number of edges of type 0 - 0 ( i.e. edge ' $a b$ ' where ' $a$ ' and ' $b$ ' both are labeled ' 0 ') be ' $x$ '

And Let number of edges of type $1-1$ be ' $y$ '

From *

Number of edges of type $0-1$ and $1-0$ are, $\alpha_{0}+\beta_{0}-2 x$

Number of edges of type $0-1$ and $1-0$ are, $\alpha_{1}+\beta_{1}-2 y$

$\Rightarrow \alpha_{0}+\beta_{0}-2 x=\alpha_{1}+\beta_{1}-2 y$

But $\alpha_{0}+\beta_{0}=\alpha_{1}+\beta_{1} \quad$ By assumption $\mathrm{G}$ is cordial

$\Rightarrow \mathrm{x}=\mathrm{y}$

But total number of edges $=4 \mathrm{~m}+2$

$\Rightarrow$ Number of edges labeled ' 0 ' $=$ Number of edges labeled ' 1 ' $\because G$ is cordial

$\Rightarrow \mathrm{x}+\mathrm{y}=2 \mathrm{~m}+1$ 
$\Rightarrow x=m+\frac{1}{2}$ and $y=m+\frac{1}{2}$

Contradiction.

Hence ' $G$ ' is not cordial for $n=4 m+2$

G IS NOT CORDIAL FOR $\mathrm{n}=4 \mathrm{~m}+2$

\section{Case IV Let $n=\mathbf{4 m + 3}$}

Consider $n=4 m+3 \quad$ for $m \in Z$

$|\mathrm{A}|=|\mathrm{B}|=4 \mathrm{~m}+3$

Let ' $\mathrm{G}$ ' be a graph $\mathrm{n}=4 \mathrm{~m}+3$

Consider any three edges $\left(u_{1}, v_{1}\right),\left(u_{2}, v_{2}\right),\left(u_{3}, v_{3}\right)$ with $u_{i} \in A$ and $v_{i} \in B$ for $\mathrm{i}=1,2,3$

Then $\mathrm{G}-u_{1}, v_{1}, u_{2}, v_{2}, u_{3}, v_{3}$ is a graph with $\mathrm{n}=4 \mathrm{~m}$

$\therefore$ By case i it has a cordial labeling with

|\# of vertices labeled ' 0 '- \# of vertices labeled ' 1 ' $\mid=0$ and

| \# of edges labeled ' 0 '- \# of edges labeled ' 1 ' $\mid=0$

Along with the same labeling label $u_{1}, v_{1}, u_{2}, v_{2}, u_{3}, v_{3}$ as follows,

$u_{1}$ as $o, v_{1}$ as $o, u_{2}$ as $o, v_{2}$ as $1, u_{3}$ as $1, v_{3}$ as 1

Then number of vertices labeled $0=$ Then number of vertices labeled 1

And labeling of $\left(u_{1}, v_{1}\right)$ is $0,\left(u_{2}, v_{2}\right)$ is $1,\left(u_{3}, v_{3}\right)$ is 0

Then number of vertices labeled $0=$ Then number of vertices labeled ' 1 ' +1

|\# of vertices labeled ' 0 '- \# of vertices labeled ' 1 ' $\mid=0$ and

| \# of edges labeled ' 0 '- \# of edges labeled ' 1 ' $\mid=1$

$\therefore$ 'G' is cordial

\section{G IS CORDIAL FOR $n=4 m+3$}

Hence the result.

Theorem 2. If $\mathrm{G}$ is a 2- regular bipartite graph with partite sets $\mathrm{A}$ and $\mathrm{B}$ with

Then $\mathrm{G}$ is cordial iff every component of $\mathrm{G}$ can be written as cycle of length $4 \mathrm{~m}$

$$
|\mathrm{A}|=|\mathrm{B}|=\mathrm{n}
$$

Let ' $G$ ' be a bipartite regular graph of degree ' 2 '.

We know that, "If ' $G$ ' is a regular bipartite graph of degree ' 2 ' then it can always be written as disjoint union of even cycles.

Let ' $G$ ' be the graph which is the cycle of length ' $2 n$ '

Claim : Cycle of length $2 \mathrm{n}$ is cordial iff $\mathrm{n}$ is even.

Part (a) : To prove: $\mathrm{n}$ is even $\Rightarrow \mathrm{G}$ is cordial.

Part (b) : To prove: $\mathrm{G}$ is cordial $\Rightarrow \mathrm{n}$ is even.

i.e. To prove: $\mathrm{n}$ is odd $\Rightarrow \mathrm{G}$ is not cordial.

Proof of (a): Consider a cycle of length $m=2 n$ where $n$ is even.

Let $\mathrm{n}=2 \mathrm{p} \Rightarrow \mathrm{m}=4 \mathrm{p}$

Let $a_{1}, a_{2}, a_{3}, a_{4}, a_{5}, \ldots \ldots a_{4 p}, a_{1}$, be the given cycle where $a_{i,}$ is adjacent to $a_{i+1}$,

For $i=1,2,3 \ldots \ldots \ldots . .4 p-1$ and $a_{4 p}$, is adjacent to $a_{1}$, 
Label $a_{1,} a_{2,} a_{3}, a_{4}, a_{5,} \ldots \ldots a_{4 p}$, as

$0,1,1,0,0,1,1,0,0,1,1, \ldots 0,1,1,0$

As $m=4 p$, we can have repeatedly $p$ times the labeling of vertices as $0,1,1,0$

Which gives edges labeling as, $1,0,1,0,1,0,1, \ldots 0$ [ last edge will be labeled ' 0 ' as

$a_{4 p}$, and $a_{1}$, both labeled 0 .

We can see that the labeling is cordial.

Hence cycle of length ' $2 \mathrm{n}$ ' where $\mathrm{n}$ is even is always cordial.

Proof of (b): Consider a cycle of length $m=2 n$ where $n$ is odd.

Let $n=2 p+1 \Rightarrow m=4 p+2$

We can write this graph as a bipartite graph with partite sets $\mathrm{A}$ and $\mathrm{B}$ where

$|\mathrm{A}|=|\mathrm{B}|=2 \mathrm{p}+1$

Let number vertices of 'A' labeled ' 0 ' be $\alpha_{0}$

Let number vertices of ' $\mathrm{A}$ ' labeled ' 1 ' be $\alpha_{1}$

Let number vertices of ' $\mathrm{B}$ ' labeled ' 0 ' be $\beta_{0}$

Let number vertices of ' $\mathrm{B}$ ' labeled ' 1 ' be $\beta_{1}$

Notation: $\alpha_{i j}{ }^{---}$number of edges 'ab' where

' $\mathrm{a}$ ' is a vertex in A labeled ' $\mathrm{i}$ ' and

' $b$ ' is a vertex in B labeled ' $\mathrm{j}$ '

With this notation we have,

$$
\begin{aligned}
& a_{11}+a_{10}=2 \alpha_{1} \\
& a_{00}+a_{01}=2 \alpha_{0} \quad \text {----------- } 2 \\
& a_{10}+a_{00}=2 \beta_{0} \quad \text {---------- } 3 \\
& a_{01}+a_{11}=2 \beta_{1} \quad \text {-------- } 4
\end{aligned}
$$

Assuming ' $\mathrm{G}$ ' is cordial we get,

$a_{11}+a_{00}=a_{10}+a_{01}-----------*$

But, $a_{11}+a_{00}+a_{10}+a_{01}=4 p+2$

$\therefore\left(a_{11}+a_{00}\right)+\left(a_{10}+a_{01}\right)=4 p+2$

From *and *

$\left(a_{11}+a_{00}\right)=2 p+1$

$\left(a_{10}+a_{01}\right)=2 p+1$

1 and $3 \Rightarrow a_{11}+a_{00}=2 \alpha_{1}+2 \beta_{0}-2 a_{10}$

$$
\Rightarrow 2 p+1=2 \alpha_{1}+2 \beta_{0}-2 a_{10}
$$

Which is a contradiction as L.H.S. is $1 \bmod 2$ and R.H.S. is $0 \bmod 2$

Hence this cycle cannot be cordial.

Hence a cycle of length $2 \mathrm{n}$ is not cordial if $\mathrm{n}$ is odd.

Thus we have proved: Cycle of length $2 \mathrm{n}$ is cordial iff $\mathrm{n}$ is even.

$\therefore$ A regular bipartite graph of degree 2 is cordial iff its every component can be written as a cycle of length $4 \mathrm{n}$.

Hence the proof. 
Theorem 3. If $\mathrm{G}$ is a n- regular bipartite graph with partite sets $\mathrm{A}$ and $\mathrm{B}$ with Then $\mathrm{G}$ is cordial

Let

$A=\left\{a_{1}, a_{2}, \ldots a_{n}\right\}$

$B=\left\{b_{1}, b_{2}, \ldots b_{n}\right\}$

Case $\mathrm{I}: \mathrm{n}$ is even

$$
\text { Let } \mathrm{n}=2 \mathrm{~m}
$$

Label $a_{1}, a_{2}, \ldots a_{n}$ as ' 0 ' and $a_{m+1}, a_{m+2}, \ldots a_{2 m}$ as ' 1 '.

For set B label any ' $m$ ' vertices as ' 0 ' any ' $m$ ' as ' 1 '

W.l.g. let $b_{1}, b_{2}, \ldots b_{n}$ are ' 0 ' and $b_{m+1}, b_{m+2}, \ldots b_{2 m}$ are ' 1 '

For the vertex $a_{1}$ :The edges incident on $a_{1}$ are,

$a_{1} b_{1}, a_{1} b_{2}, \ldots a_{1} b_{m}, a_{1} b_{m+1}, a_{1} b_{m+2}, \ldots a_{1} b_{2 m}$, out of which,

$a_{1} b_{1}, a_{1} b_{2}, \ldots a_{1} b_{m}$ are labeled ' 0 ' and $a_{1} b_{m+1}, a_{1} b_{m+2}, \ldots a_{1} b_{2 m}$, are labeled ' 1 '.

Which gives equal number of edges labeled 0 and 1 each equal to ' $m$ '

Similarly for remaining vertices.

Hence we have in all $2 m^{2}$ edges labeled o and $2 m^{2}$ edges labeled 1 .

$\therefore \mid \#$ of edges labeled ' 0 '- \# of edges labeled ' 1 ' $\mid=1$

$\therefore \mathrm{G}^{\prime}$ is cordial

Case II $: \mathrm{n}$ is odd

Let $\mathrm{n}=2 \mathrm{~m}+1$

Consider $\mathrm{A}-\{\mathrm{u}\}$ and $\mathrm{B}-\{\mathrm{v}\}$ for some $\mathrm{uv} \in \mathrm{V}(\mathrm{G})$

Then by case $(i)$ it has a cordial labeling with

$2 m^{2}$ edges labeled 0 and $2 m^{2}$ edges labeled 1

Now label $\mathrm{u}$ as 0 and $\mathrm{v}$ as 1

As it is complete bipartite we have edges,

$u b_{1}, u b_{2}, \ldots u b_{m}, u b_{m+1}, u b_{m+2}, \ldots u b_{2 m}, u v$ which are labeled as

$0,0, \ldots 0,1, \quad 1, \ldots .1, \quad 1$ respectively giving $\mathrm{m}$ edges labeled 0 and $\mathrm{m}+1$ edges labeled 1 .

Also, $a_{1} v, a_{2} v, \ldots a_{m} v, a_{m+1} v, a_{m+2} v, \ldots ., a_{2 m} v \quad$ which are labeled as

$1,1, \ldots \quad 1, \quad 0, \quad 0, \ldots . \quad 0$ respectively giving $\mathrm{m}$ edges labeled 0 and $\mathrm{m}$ edges labeled 1.

$\therefore$ In all we get, Number of edges labeled $0=2 m^{2}+2 m$ and

Number of edges labeled $1=2 m^{2}+2 m+1$

| \# of edges labeled ' 0 '- \# of edges labeled ' 1 ' $\mid=1$

$\therefore$ 'G' is cordial

Hence the proof.

Theorem 4. If $\mathrm{G}$ is a $\mathrm{n}$ - regular bipartite graph with partite sets $\mathrm{A}$ and $\mathrm{B}$ with

Then $\mathrm{G}$ is cordial iff $\mathrm{n}=0,1 \bmod 4$.

$$
|\mathrm{A}|=|\mathrm{B}|=\mathrm{n}
$$

Case (i ) : $\mathrm{n}=4 \mathrm{~m}$

Let $\mathrm{A}=\left\{a_{1}, a_{2}, \ldots, a_{n}\right\}$ and $\mathrm{B}=\left\{b_{1}, b_{2}, \ldots b_{n}\right\}$

And $\mathrm{G}$ is a (k-1) bipartite graph with partite sets A,B.

$\therefore$ It can be obtained by removing one and only one edge of every vertex of a complete graph.

Label $a_{1}, a_{2}, \ldots a_{2 m}$ as 0 and $a_{2 m+1}, a_{2 m+2}, \ldots a_{4 m}$ as 1

Then we can have ' $\mathrm{G}$ ' is a graph obtained by removing

$a_{1} b_{i_{1}}, a_{2} b_{i_{2}}, \ldots . ., a_{4 m} b_{i_{4 m}}$ where $b_{i_{j}} \neq b_{i_{k}}$ for $i_{j} \neq i_{k}$ from the complete bipartite graph.

We will rename the vertices in the set ' $\mathrm{B}$ ' such that the edges removes are,

$a_{1} b_{1}, a_{2} b_{2}, \ldots a_{4 m} b_{4 m}$ 
Now label $b_{1}, b_{2}, \ldots b_{4 m}$ as,

$b_{1}, b_{2}, \ldots b_{m}$ as ' 0 ', $b_{m+1}, b_{m+2}, \ldots b_{2 m}$ as ' 1 ' $, b_{2 m+1}, b_{2 m+2}, \ldots b_{3 m}$ as ' 0 ', $b_{3 m+1}, b_{3 m+2}, \ldots b_{4 m}$ as ' 1 ',

Then the edges removed from the complete graph are labeled as,
$a_{1} b_{1}, a_{2} b_{2}, \ldots a_{m} b_{m}$
$0,0, \ldots 0$ total ' $m$ ' edges
$a_{m+1} b_{m+1}, a_{m+2} b_{m+2}, \ldots a_{2 m} b_{2 m}$
$1,1, \ldots 1$ total 'm' edges
$a_{2 m+1} b_{2 m+1}, a_{2 m+2} b_{2 m+2}, \ldots a_{3 m} b_{3 m}$
$0,0, \ldots 0$ total ' $m$ ' edges
$a_{3 m+1} b_{3 m+1}, a_{3 m+2} b_{3 m+2}, \ldots a_{4 m} b_{4 m}$ total ' $m$ ' edges

$\therefore$ For ' $G$ ' Number of vertices labeled $0=$ number of vertices labeled $1=4 \mathrm{~m}$

And Number of edges labeled $0=$ number of edges labeled $1=8 m^{2}-2 m$

( from the case $\mathrm{k}=\mathrm{n}$ )

$\therefore \mid$ \# of vertices labeled ' 0 '- \# of vertices labeled ' 1 ' $\mid=0$

and

| \# of edges labeled ' 0 '- \# of edges labeled ' 1 ' $\mid=0$

$\therefore$ ' $G$ ' is cordial

Case (ii) : $n=4 m+1$

As $\mathrm{G}$ is a (n-1)-regular bipartite graph, with the partitions $\mathrm{A}$ and $\mathrm{B}$

Where $\mathrm{A}=\left\{a_{1}, a_{2}, \ldots, a_{n}\right\}$ and $\mathrm{B}=\left\{b_{1}, b_{2}, \ldots b_{n}\right\}$

By case ( $\mathrm{i}$ ) with the similar arguments let $\mathrm{G}$ is obtained from the complete bipartite graph by deleting the edges,

$a_{1} b_{1}, a_{2} b_{2}, \ldots a_{4 m} b_{4 m}, a_{4 m+1} b_{4 m+1}$ Where,

$a_{1}, a_{2}, \ldots a_{2 m}, a_{2 m+1}$ are labeled as 0 and $a_{2 m+2}, a_{2 m+2}, \ldots a_{4 m+1}$ as 1 .

Now label the vertices of $\mathrm{B}$ as follows.

$b_{1}, b_{2}, \ldots b_{m}$ as ' 0 ', $b_{m+1}, b_{m+2}, \ldots b_{2 m}, b_{2 m+1}$ as ' 1 ', $b_{2 m+2}, b_{2 m+2}, \ldots b_{3 m+1}$ as ' 0 ', $b_{3 m+2}, b_{3 m+2}, \ldots b_{4 m+1}$ as ' 1 ',

The edges removed from the complete graph and there labelings are as follows :

$a_{1} b_{1}, a_{2} b_{2}, \ldots a_{m} b_{m} \quad 0,0, \ldots 0 \_$total 'm' edges

$a_{m+1} b_{m+1}, a_{m+2} b_{m+2}, \ldots a_{2 m} b_{2 m}, a_{2 m+1} b_{2 m+1} \ldots 1,1, \ldots 1 \_$total 'm+1' edges

$a_{2 m+2} b_{2 m+2}, a_{2 m+3} b_{2 m+3}, . ., a_{3 m+1} b_{3 m+1} \ldots 1,1, \ldots 1 \_$total ' $\mathrm{m}$ ' edges

$a_{3 m+2} b_{3 m+2}, a_{3 m+3} b_{3 m+3}, \ldots a_{4 m+1} b_{4 m+1} \ldots 0,0, \ldots 0 \_$total 'm' edges

$\therefore$ Number of edges labeled 1 are $8 m^{2}+4 m+1-(m+1)-m$

$$
=8 m^{2}+2 m
$$

Number of edges labeled 0 are $8 m^{2}+4 m-m-m$

$$
=8 m^{2}+2 m
$$

$\therefore$ Number of edges labeled $1=$ Number of edges labeled 0

Hence $\mathrm{G}$ is cordial.

$(n-1)-$ regular graph is cordial for $n=4 m+1$

Case ( iii ) : $\mathrm{n}=4 \mathrm{~m}+2$

As $\mathrm{G}$ is a (n-1)-regular bipartite graph, with the partitions $\mathrm{A}$ and $\mathrm{B}$

Where $\mathrm{A}=\left\{a_{1}, a_{2}, \ldots, a_{n}\right\}$ and $\mathrm{B}=\left\{b_{1}, b_{2}, \ldots b_{n}\right\}$ 
$\therefore$ Number of edges in G is $(4 m+2)(4 m+1)=16 m^{2}+12 m+2$

Let $\alpha_{0}$ be number of vertices in A labeled ' 0 ' $\alpha_{1}$ be number of vertices in A labeled ' 1 ' $\beta_{0}$ be number of vertices in B labeled ' 0 ' $\beta_{1}$ be number of vertices in $\mathrm{B}$ labeled ' 1 '

$\therefore$ Total number of edges 'ab' where 'a' is labeled 0 and $b \in B=\alpha_{0}(4 m+1)$

Total number of edges 'ab' where ' $\mathrm{b}$ ' is labeled 0 and $a \in A=\beta_{0}(4 m+1)$

Total number of edges 'ab' where 'a' is labeled 1 and $b \in B=\alpha_{1}(4 m+1)$

Total number of edges 'ab' where ' $\mathrm{b}$ ' is labeled 1 and $a \in A=\beta_{1}(4 m+1)$

Let Total number of edges ' $a b$ ' where ' $a$ ' and ' $b$ ' both are labeled $0=x$

Total number of edges ' $a b$ ' where ' $a$ ' and ' $b$ ' both are labeled $1=y$

(i.e. $0-0$ and $1-1$ type)

$\therefore$ The number of edged 'ab' of labeling $0 \& 1$ And $1 \& 0$ are

$\alpha_{0}(4 m+1)+\beta_{0}(4 m+1)-x \quad$ Also,

The number of edged 'ab' of labeling $1 \& 0$ And 0\&01are

$\alpha_{1}(4 m+1)+\beta_{1}(4 m+1)-y$

$\therefore \alpha_{0}(4 m+1)+\beta_{0}(4 m+1)-x=\alpha_{1}(4 m+1)+\beta_{1}(4 m+1)-y$

$\Rightarrow\left(\alpha_{0}+\beta_{0}\right)(4 m+1)-2 x=\left(\alpha_{1}+\beta_{1}\right)(4 m+1)-2 y$

But, $\left(\alpha_{0}+\beta_{0}\right)=\left(\alpha_{1}+\beta_{1}\right)$ as $\mathrm{G}$ is cordial

$\Rightarrow \mathrm{x}=\mathrm{y}$

Now as total number of edges are $16 m^{2}+12 m+2$

number of edges labeled $0=8 m^{2}+6 m+1$

$\Rightarrow \mathrm{x}+\mathrm{y}=8 m^{2}+6 m+1 \Rightarrow 2 \mathrm{x}=8 m^{2}+6 m+1 \quad \because x=y$ as $G$ is cordial

Contradiction $\quad \because$ L.H.S. is even \& R.H.S. is odd

Hence G is Not cordial.

\section{(n-1) -regular graph is not cordial for $n=4 m+2$}

Case (iii) : $n=4 m+3$

Total number of edges $=(4 m+3)(4 m+2)=16 m^{2}+20 m+6$

Using the same notations and the same arguments as in case ( iii ) we get,

$\therefore \alpha_{0}(4 m+2)+\beta_{0}(4 m+2)-x=\alpha_{1}(4 m+2)+\beta_{1}(4 m+2)-y$

$\Rightarrow\left(\alpha_{0}+\beta_{0}\right)(4 m+2)-2 x=\left(\alpha_{1}+\beta_{1}\right)(4 m+2)-2 y$

But, $\left(\alpha_{0}+\beta_{0}\right)=\left(\alpha_{1}+\beta_{1}\right)$ as $\mathrm{G}$ is cordial

$\Rightarrow \mathrm{x}=\mathrm{y}$

Now ,as total number of edges are $16 m^{2}+20 m+6$

number of edges labeled $0=8 m^{2}+10 m+3$ 


$$
\begin{aligned}
& \Rightarrow \mathrm{x}+\mathrm{y}=8 m^{2}+10 m+3 \\
& \Rightarrow 2 \mathrm{x}=8 m^{2}+10 m+3 \quad \because x=y \text { as Gis cordial } \\
& \text { Contradiction } \because \text { L.H.S. is even \& R.H.S. is odd }
\end{aligned}
$$

\section{Hence G is NOT cordial}

(n-1)-regular graph is not cordial for $n=4 m+3$

Hence the proof.

\section{Conclusion}

- If $\mathrm{G}$ is a 1-regular bipartite graph with partite sets ' $\mathrm{A}$ ' and 'B' such that $|\mathrm{A}|=|\mathrm{B}|=\mathrm{n}$ then ' $\mathrm{G}$ ' is cordial iff $\mathrm{n}=0,1,3 \bmod 4$

- If $G$ is a 2-regular bipartite graph with partite sets ' $A$ ' and 'B' such that $|A|=|B|=n$ then ' $G$ ' is cordial iff its every component is a cycle of length $4 \mathrm{~m}$.

- If $\mathrm{G}$ is a n-regular $n \geq 3$ bipartite graph with partite sets 'A' and 'B' such that $|\mathrm{A}|=|\mathrm{B}|=\mathrm{n}$ then ' $\mathrm{G}$ ' is cordial.

- If $\mathrm{G}$ is a $n-1$-regular bipartite graph with partite sets 'A' and ' $\mathrm{B}$ ' such that $|\mathrm{A}|=|\mathrm{B}|=\mathrm{n}$ then ' $\mathrm{G}$ ' is cordial iff $n=0,1 \bmod 4$.

\section{References}

[1] L.CAI,X.ZHU, (2001), "Gaming coloring index of graphs", Journal of graph theory, 36, 144-155

[2] XUDING ZHU, (1999), "The game coloring number of planar graphs", Journal of Combinatorial Theory Series B, 75, 245-258

[3] H. YEH, XUDING ZHUING, (2003), "4-colorable, 6-regular toroidal graphs", Discrete Mathematics, 273, 1-3,261-274

[4] X.ZHU, (2006), "Recent development in circular coloring of graphs", Topics in discrete mathematics, spinger 5, 497-550

[5] H.CHANG, X.ZHU, (2008), "Coloring games on outerplanar graphs \& trees", Discrete mathematics,doi10.1016/j.disc, 9-15

[6] H. HAJIABLOHASSAN, X. ZHU, (2003),: The circular chromatic number and mycielski construction”, Journal of graph theory,404, 106-115 